\title{
Myocardial Perfusion in Nonischemic Dilated Cardiomyopathy With and Without Atrial Fibrillation
}

Felix T. Range*1-3, Matthias Paul ${ }^{* 2,3}$, Klaus P. Schäfers ${ }^{1}$, Tayfun Acil ${ }^{2}$, Peter Kies ${ }^{1,3}$, Sven Hermann ${ }^{1}$, Otmar Schober ${ }^{1}$, Günter Breithardt ${ }^{2}$, Thomas Wichter ${ }^{2,3}$, and Michael A. Schäfers ${ }^{1,3}$

${ }^{1}$ Department of Nuclear Medicine, University of Münster, Münster, Germany; ${ }^{2}$ Department of Cardiology and Angiology, University of Münster, Münster, Germany; and ${ }^{3}$ Interdisciplinary Centre of Clinical Research (IZKF), University of Münster, Münster, Germany

Recent studies have shown that idiopathic atrial fibrillation (AF) is associated with diminished myocardial perfusion and perfusion reserve, which are also impaired in various forms of cardiomyopathies. In many cases, AF develops during progression of dilated cardiomyopathy (DCM) and may aggravate heart failure. This study compared myocardial perfusion between patients with nonischemic DCM with and without AF. Methods: Twelve men (age $\pm S D, 55 \pm 12$ y) who had DCM and persistent AF were compared with a group of 18 men (mean age, $43 \pm 15 \mathrm{y}$, $P=$ not statistically significant) who had DCM and sinus rhythm and with 22 healthy controls (mean age, $47 \pm 13$ y, $P=$ not statistically significant). Myocardial blood flow (MBF) was noninvasively quantified at rest and during adenosine infusion using $\mathrm{PET}$ and radioactive-labeled water $\left(\mathrm{H}_{2}{ }^{15} \mathrm{O} \mathrm{PET}\right)$. Results: Compared with controls, DCM patients without AF showed impaired hyperemic perfusion $(2.52 \pm 1.29$ vs. $3.57 \pm 0.88 \mathrm{~mL} / \mathrm{min} / \mathrm{mL}$, $P=0.014)$ and perfusion reserve $(2.10 \pm 1.01$ vs. $3.37 \pm 0.97$, $P=0.003)$. However, compared with DCM patients without AF, DCM patients with AF showed an additional impairment in resting perfusion $(0.82 \pm 0.31 \mathrm{~mL} / \mathrm{min} / \mathrm{mL}, P=0.010)$ and hyperemic perfusion (1.32 $\pm 0.93 \mathrm{~mL} / \mathrm{min} / \mathrm{mL}, P=0.022$ ), and compared with controls, DCM patients with AF showed a further diminishment of perfusion reserve $(1.68 \pm 0.94$ vs. $3.37 \pm 0.97$, $P<0.001)$ accompanied by the highest coronary vascular resistance of all groups. Conclusion: Compared with patients with sinus rhythm, patients with AF have significantly reduced myocardial perfusion reserve and increased coronary resistance in nonischemic DCM. Further studies on the underlying pathomechanisms are warranted.

Key Words: atrial fibrillation; dilated cardiomyopathy; myocardial blood flow; positron emission tomography

J Nucl Med 2009; 50:390-396

DOI: 10.2967/jnumed.108.055665

\footnotetext{
A trial fibrillation (AF) is the most common sustained arrhythmia in humans. It frequently requires medical treat-

Received Jul. 3, 2008; revision accepted Dec. 5, 2008.

For correspondence or reprints contact: Michael A. Schäfers, Department of Nuclear Medicine, Albert-Schweitzer-Strasse 33, D-48149, Münster, Germany.

E-mail: schafmi@uni-muenster.de

${ }^{*}$ Contributed equally to this work.

COPYRIGHT ๑ 2009 by the Society of Nuclear Medicine, Inc.
}

ment and is associated with increased morbidity and mortality (1). We have recently shown that idiopathic AF is associated with diminished myocardial perfusion and perfusion reserve (2). However, AF is more likely to develop on the basis of a structural heart disease and occurs in more than a quarter of cases with dilated cardiomyopathy (DCM) (3). Many patients with nonischemic DCM, as well as patients with idiopathic AF, present with symptoms suggestive of myocardial ischemia, such as unspecific retrosternal sensations resembling angina pectoris, despite exclusion of coronary artery disease. In nonischemic DCM, elevated markers of myocardial damage have been reported after cardiac decompensation (4). AF on top of an existing cardiomyopathy further impairs both cardiac function and individual prognosis and leads to an increased number of hospitalizations due to congestive heart failure (5). Restoration of sinus rhythm (SR) in patients with congestive heart failure and AF may ameliorate ventricular function and may spare heart transplantation (6). Myocardial perfusion and perfusion reserve are known to be impaired in various forms of cardiomyopathy (ischemic and nonischemic) even without clinically overt congestive heart failure (7).

On the basis of these facts, we hypothesized that myocardial perfusion and perfusion reserve could be less in patients with nonischemic DCM and additional AF than in patients with DCM in SR. This impairment could have prognostic and future therapeutic implications, especially since the level of hyperemic perfusion has been shown to be an independent predictor of prognosis in patients with DCM (8). Therefore, our study investigated the difference in myocardial perfusion and perfusion reserve in patients with nonischemic DCM with and without AF using noninvasive PET and radioactively labeled water $\left(\mathrm{H}_{2}{ }^{15} \mathrm{O} \mathrm{PET}\right)$.

\section{MATERIALS AND METHODS}

\section{Study Population}

Patients. A total of 30 male DCM patients were enrolled in this study. DCM was clinically diagnosed on the basis of reduced ventricular function (ejection fraction $<55 \%$ ) with left ventricular dilatation in the absence of coronary artery disease (9). Coronary 
artery disease was excluded on the basis of absence of clinical symptoms suggestive of ischemia, absence of signs of ischemia in exercise electrocardiograms, and additional coronary angiography studies in 28 of 30 DCM patients and stress myocardial SPECT studies in the remaining 2 of 30 . Further exclusion criteria were other functional cardiac diseases, hyperthyroidism, and any grade of valvular stenosis except for valvular regurgitation lower than grade 2 .

Within the DCM group, 12 patients (mean age, $55 \pm 12$ y) had persistent $\mathrm{AF}$ (mean duration, $32 \pm 39 \mathrm{mo}$ ) (DCM/AF group), whereas the remaining 18 patients (mean age, $43 \pm 15 \mathrm{y}, P=$ not statistically significant [NS] vs. DCM/AF) showed stable SR and no history of AF (DCM/SR group). None of these patients was included in our prior study (2) of patients with idiopathic AF and otherwise healthy hearts, and there is no overlap between these study groups.

Three patients in the DCM/AF group and 5 patients in the DCM/SR group had implanted cardioverter-defibrillators because of a history of life-threatening tachyarrhythmias. None of the devices worked in pacemaker mode during the scan or beforehand, as ensured by interrogation of the device.

Most patients in both DCM groups were in New York Heart Association functional class II or III (2.06 \pm 1.07 for DCM/SR vs. $2.40 \pm 0.52$ for DCM/AF, $P=\mathrm{NS}$ ).

Echocardiography was performed on all patients by expert investigators to assess atrial and ventricular dimensions and ventricular and valvular function (Table 1). These parameters were quantified in several consecutive measurements in diastole for an average $\mathrm{R}-\mathrm{R}$ interval as measured by continuous monitoring during echocardiography. This technique is in line with former study protocols (10).

Between the DCM/SR and DCM/AF groups, echocardiographically assessed left ventricular diameters, and function did not show any significant difference. As expected, the diameter of the left atrium was larger in the DCM/AF patients than in the DCM/ SR patients and controls.
The clinical characteristics of DCM/AF and DCM/SR patients are detailed in Table 1; echocardiographic parameters are listed in Table 2.

Control Subjects. Twenty-two healthy male control subjects (mean age, $47 \pm 13 \mathrm{y}, P=\mathrm{NS}$ vs. DCM/AF and DCM/SR) who had SR were enrolled. Coronary artery disease was excluded as described above and additionally by invasive angiography in 13 of 22 subjects (59\%), proving that there were no signs of coronary artery disease. All control subjects underwent maximal exercise stress testing before inclusion in the study, without any pathologic findings. Clinical and echocardiographic data are given in Tables 1 and 2.

\section{Study Design}

Noninvasive Measurement of Myocardial Perfusion In Vivo. PET scans were obtained after the subjects had fasted for at least $12 \mathrm{~h}$, particularly excluding caffeine-containing beverages, chocolate, and smoking to avoid any interference with adenosine. All medications were withheld for $24 \mathrm{~h}$ before imaging. All scans were initiated between 8:00 and 9:00 AM, assuring the same circadian conditions in all participants. Before baseline measurements, all study participants rested supine on the scanner for $15 \mathrm{~min}$.

The study followed a protocol previously published (2). Briefly, myocardial blood flow (MBF) was assessed by dynamic PET (ECAT-921; Siemens/CTI) after an intravenous bolus injection of $500 \mathrm{MBq}$ of $\mathrm{H}_{2}{ }^{15} \mathrm{O}$ over $20 \mathrm{~s}$. A 26-frame dynamic PET acquisition was obtained over $5 \mathrm{~min}$. The emission data were reconstructed (Hanning filter, $7.3 \mathrm{~mm}$ in full width at half maximum, zoom factor of $2.3,47$ planes, and matrix size of $128 \times 128$ ). Factor images were generated from the dynamic $\mathrm{H}_{2}{ }^{15} \mathrm{O}$ scans and resliced into short-axis images perpendicular to the long axis of the left ventricle. This transformation matrix was also used for reslicing the dynamic water images. Regions of interest (ROIs) were placed manually on the short-axis planes of the factor images

\begin{tabular}{|c|c|c|c|c|c|c|}
\hline \multirow[b]{2}{*}{ Parameter } & \multicolumn{2}{|c|}{ DCM/AF patients } & \multicolumn{2}{|c|}{ DCM/SR patients } & \multicolumn{2}{|c|}{ Control subjects } \\
\hline & Mean $\pm S D$ & $\bar{n}$ & Mean $\pm S D$ & $n$ & Mean $\pm S D$ & $\bar{n}$ \\
\hline Age $(y)$ & $55 \pm 12$ & 12 & $43 \pm 15$ & 18 & $47 \pm 13$ & 22 \\
\hline AF duration (mo) & $32 \pm 39$ & 9 & - & - & - & - \\
\hline NYHA class & $2.4 \pm 0.5$ & 10 & $2.1 \pm 1.1$ & 17 & 1 & 22 \\
\hline \multicolumn{7}{|l|}{ Risk factors (\%) } \\
\hline Hypertension & 42 & 12 & 22 & 18 & 41 & 22 \\
\hline Diabetes mellitus & 33 & 12 & 22 & 18 & 0 & 22 \\
\hline Smoking & 42 & 12 & 33 & 18 & 27 & 22 \\
\hline Family history & 17 & 12 & 39 & 18 & 18 & 22 \\
\hline Hypercholesterolemia & 58 & 12 & 29 & 17 & 45 & 22 \\
\hline Obesity & 58 & 12 & 22 & 18 & 23 & 22 \\
\hline Framingham risk score & $13 \pm 7$ & 12 & $8 \pm 8$ & 15 & $9 \pm 8$ & 19 \\
\hline \multicolumn{7}{|l|}{ Medication } \\
\hline Amiodarone & $42^{*}$ & 12 & 6 & 18 & 0 & 22 \\
\hline ACE inhibitor/AT1 antagonist & $100^{\star+}$ & 12 & $89^{+}$ & 18 & 9 & 22 \\
\hline$\beta$-receptor antagonist & $92^{\star \dagger}$ & 12 & 61 & 18 & 32 & 22 \\
\hline Calcium antagonist & 0 & 12 & 6 & 18 & 5 & 22 \\
\hline Digitalis & $92^{*}$ & 12 & 22 & 18 & 0 & 22 \\
\hline $\begin{array}{l}{ }^{\star} P<0.05 \text { vs. DCM/SR patients } \\
{ }^{\dagger} P<0.05 \text { vs. controls. } \\
\text { NYHA }=\text { New York Heart Asso }\end{array}$ & & & & & & \\
\hline
\end{tabular}


TABLE 2. Echocardiographic Parameters

\begin{tabular}{|c|c|c|c|}
\hline Parameter & $\begin{array}{l}\mathrm{DCM} / \mathrm{AF} \\
\text { patients }\end{array}$ & $\begin{array}{l}\mathrm{DCM} / \mathrm{SR} \\
\text { patients }\end{array}$ & Controls \\
\hline$n$ & 12 & 18 & 22 \\
\hline LA (cm) & $5.4 \pm 1.0^{* \dagger}$ & $4.0 \pm 1.2$ & $3.9 \pm 0.5$ \\
\hline LV EDD (cm) & $6.5 \pm 1.0$ & $6.3 \pm 0.8$ & $5.4 \pm 0.8$ \\
\hline LV ESD (cm) & $4.5 \pm 1.2^{*}$ & $4.7 \pm 0.8^{*}$ & $3.3 \pm 0.5$ \\
\hline PW (cm) & $1.2 \pm 0.2^{*}$ & $1.0 \pm 0.1$ & $1.1 \pm 0.1$ \\
\hline IVS (cm) & $1.2 \pm 0.2$ & $0.9 \pm 0.1^{*}$ & $1.2 \pm 0.2$ \\
\hline FS (\%) & $25.2 \pm 10.8^{*}$ & $24.3 \pm 5.2^{*}$ & $39.1 \pm 5.6$ \\
\hline LV EF (\%) & $36.7 \pm 12.4^{*}$ & $31.7 \pm 14.4^{*}$ & $66.9 \pm 5.6$ \\
\hline \multicolumn{4}{|c|}{$\begin{array}{l}{ }^{*} P<0.05 \text { vs. controls. } \\
{ }^{\dagger} P<0.05 \text { vs. DCM/SR patients. } \\
\text { LA and LV = left atrial and left ventricular diameters, } \\
\text { espectively; EDD and ESD = end-diastolic and end-systolic } \\
\text { diameters, respectively; PW and IVS = posterior wall and } \\
\text { nterventricular septum thicknesses, respectively; FS = frac- } \\
\text { ional shortening; EF = ejection fraction. }\end{array}$} \\
\hline
\end{tabular}

encompassing left ventricular myocardial tissue, the left atrial cavity, and the right ventricular cavity. The myocardial ROIs covered the whole left ventricular myocardium since these were drawn on all short-axis images showing myocardium, typically 11-12 slices. In addition to the assessment of a single ROI comprising the whole left ventricular myocardium, separate values were obtained for 4 ROIs by dividing the myocardial tissue ROI into an anterior, lateral, inferior, and septal region.

Arterial, venous, and tissue time-activity curves were fitted to a single-compartment model to quantify regional and global MBF $(\mathrm{mL} / \mathrm{min} / \mathrm{mL})$ and perfusable tissue fraction (milliters of waterperfused tissue per milliliter of ROI) (11).

All patients and control subjects gave written informed consent to the study protocol, which was approved by the Ethics Committee of the Medical faculty of the University of Münster and the Chamber of Physicians (Ärztekammer Westfalen-Lippe), Münster, Germany.

\section{Hyperemic Flow and Flow Reserve}

Additionally, hyperemic MBF was measured from a second injection of $\mathrm{H}_{2}{ }^{15} \mathrm{O} 2 \mathrm{~min}$ after initiation of a 7-min adenosine infusion at $140 \mu \mathrm{g} / \mathrm{kg}$ of body weight per minute. The hyperemic coronary flow reserve was calculated as the ratio of hyperemic and baseline MBF, the latter represented by the MBF corrected for the rate-pressure product (RPP). To allow for sufficient decay of the radioactivity from the first PET scan, the adenosine PET scan started $20 \mathrm{~min}$ after the end of the resting scan.

\section{Hemodynamics}

Heart rate and systolic, diastolic, and mean arterial blood pressures were determined for each subject during PET scans. Because of the unreliability of heart rate detection by a bedside electrocardiography monitor in AF, caused by beat-to-beat R-R interval changes, mean heart rates and variance of cycle length were calculated from continuous Holter recordings during the scans (R-R intervals SD of normal to normal).

\section{Coronary Vascular Resistance (CVR)}

CVR in the different settings (baseline and adenosine) was calculated by dividing mean arterial pressure by the respective MBF.

\section{Statistical Analysis}

Results are expressed as mean $\pm \mathrm{SD}$. After testing for the equality of variances (Levene test), ANOVA in connection with the Bonferroni correction was performed for comparisons of global values of MBF, CVR, and coronary flow reserve between groups. The Pearson correlation coefficient was calculated to investigate associations between possible confounders and measured data of perfusion, perfusion reserve, and CVR. A $P$ value of less than 0.05 was considered significant.

\section{RESULTS}

\section{Hemodynamics}

Data on hemodynamics are given in Table 3. To eliminate potential interindividual differences in cardiac workload that could influence $\mathrm{MBF}, \mathrm{MBF}$ at rest was corrected for the ratepressure product $\left(\mathrm{MBF}_{\text {corrected }}=\mathrm{MBF} / \mathrm{RPP} \times 10,000\right)(12)$. Additionally, all values of uncorrected $\mathrm{MBF}$ at baseline are also listed in the text and in Figure 1. Adenosine-induced MBF remained uncorrected because the MBF increase stimulated by adenosine is grossly independent of RPP (13).

\section{MBF and Flow Reserve}

DCM/SR Versus Controls. As expected from previously published studies (14), resting MBF corrected for RPP was comparable in DCM/SR patients and controls (1.13 $\pm 0.31 \mathrm{vs.}$ $1.14 \pm 0.20 \mathrm{~mL} / \mathrm{min} / \mathrm{mL}, P=\mathrm{NS})$. These similarities were consistently found in MBF values uncorrected for the ratepressure product $(0.84 \pm 0.27$ vs. $0.87 \pm 0.16 \mathrm{~mL} / \mathrm{min} / \mathrm{mL}$, $P=$ NS) (Fig. 1). In contrast, hyperemic MBF (2.52 \pm 1.29 vs. $3.57 \pm 0.88 \mathrm{~mL} / \mathrm{min} / \mathrm{mL}, P=0.014)$ and hyperemic coronary flow reserve $(2.10 \pm 1.01$ vs. $3.37 \pm 0.97, P=0.003)$ were significantly impaired in DCM/SR patients (Figs. 1 and 2), compared with controls.

$D C M / A F$ Versus DCM/SR. Interestingly, baseline MBF corrected for RPP was significantly lower in DCM/AF patients $(0.82 \pm 0.31 \mathrm{~mL} / \mathrm{min} / \mathrm{mL}, P=0.010)$ than in DCM/SR patients. Accordingly, uncorrected baseline $\mathrm{MBF}$ was also lower in DCM/AF patients and significantly less than in controls $(0.67 \pm 0.20 \mathrm{~mL} / \mathrm{min} / \mathrm{mL}, P=0.038$ vs. controls $)$. Even more pronounced, hyperemic $\mathrm{MBF}$ was further impaired in DCM/AF patients $(1.32 \pm 0.93 \mathrm{~mL} / \mathrm{min} / \mathrm{mL}, P=0.022)$ (Fig. 1). As a consequence, DCM/AF patients showed an almost blunted hyperemic coronary flow reserve (1.68 \pm $0.94 \mathrm{~mL} / \mathrm{min} / \mathrm{mL}, P=\mathrm{NS}$ ) (Fig. 2).

\section{Regional MBF}

In addition to these global analyses of $\mathrm{MBF}$, regional MBF in 4 ROIs (anterior, lateral, inferior, and septal walls) was analyzed. Myocardial perfusion was homogeneously distributed throughout the left ventricular myocardium in all groups. None of the regions showed a perfusion significantly different from any other region within any single group. Global perfusion differences between groups did not show any dependency on specific regional distribution patterns. Table 4 contains a detailed list of regional $\mathrm{MBF}$ values. 


\begin{tabular}{|c|c|c|c|c|c|c|}
\hline \multirow[b]{2}{*}{ Parameter } & \multicolumn{3}{|c|}{ Baseline } & \multicolumn{3}{|c|}{ Adenosine } \\
\hline & DCM/AF & DCM/SR & Controls & DCM/AF & DCM/SR & Controls \\
\hline$n$ & 12 & 18 & 22 & 10 & 14 & 22 \\
\hline SBP & $131 \pm 25^{\star}$ & $110 \pm 16$ & $125 \pm 18$ & $128 \pm 20$ & $116 \pm 13$ & $130 \pm 16$ \\
\hline DBP & $75 \pm 10$ & $68 \pm 12$ & $76 \pm 8$ & $72 \pm 12$ & $66 \pm 13$ & $73 \pm 9$ \\
\hline MAP & $94 \pm 13^{*}$ & $82 \pm 12$ & $92 \pm 11$ & $91 \pm 13$ & $83 \pm 12$ & $92 \pm 10$ \\
\hline HR & $66 \pm 11$ & $68 \pm 8$ & $62 \pm 8$ & $67 \pm 14^{\dagger}$ & $78 \pm 15$ & $91 \pm 14$ \\
\hline RPP & $8,520 \pm 1,865$ & $7,476 \pm 1,307$ & $7,801 \pm 1,743$ & $8,552 \pm 2,165^{\dagger}$ & $9,056 \pm 2,221$ & $11,924 \pm 2,336$ \\
\hline
\end{tabular}

\section{CVR}

DCM/SR Versus Controls. CVR was similar in DCM/SR patients and controls at baseline (106 \pm 34 vs. $109 \pm 21$ $\left.\mathrm{mm} \mathrm{Hg} \times \mathrm{mL}^{-1} \times \min \times \mathrm{mL}, P=\mathrm{NS}\right)$ and under adenosine infusion ( $49 \pm 51$ vs. $27 \pm 7 \mathrm{~mm} \mathrm{Hg} \times \mathrm{mL}^{-1} \times \min \times \mathrm{mL}$, $P=$ NS) (Fig. 3).

$D C M / A F$ Versus DCM/SR. In DCM patients, the presence of AF dramatically increased CVR at baseline (150 \pm $\left.40 \mathrm{~mm} \mathrm{Hg} \times \mathrm{mL}^{-1} \times \min \times \mathrm{mL}, P=0.001\right)$ and under adenosine infusion $\left(95 \pm 45 \mathrm{~mm} \mathrm{Hg} \times \mathrm{mL}^{-1} \times \min \times \mathrm{mL}\right.$, $P=0.013)$ (Fig. 3).

\section{Clinical Characteristics and Potentially Confounding Factors}

Age and Sex. Only male subjects were studied. The mean age of DCM/AF patients was not significantly different from that of DCM/SR patients.

Risk Factors. The DCM groups did not significantly differ with respect to any single coronary risk factor (total cholesterol, high LDL cholesterol, low HDL cholesterol, hypertension, diabetes, smoking, or family history of cor- onary artery disease) or the Framingham score assessing the individual global cardiovascular risk. No correlation between these risk factors or the risk score and PET data was found within any group.

\section{DISCUSSION}

$\mathrm{AF}$ in the presence of structural heart disease is a frequent clinical problem (3) that may severely affect the patients' quality of life and cardiac function $(5,6)$. In a recent study, we found impaired myocardial perfusion and perfusion reserve in idiopathic AF (2). Therefore, the aim of this study was to investigate whether the presence of $\mathrm{AF}$ is also associated with impairment of myocardial perfusion in patients with nonischemic DCM. We have used state-of-the-art PET technology uniquely able to quantify myocardial perfusion and perfusion reserve in vivo in DCM patients with SR or persistent AF. The main and new findings of this study are that, compared with DCM patients with preserved SR, patients with DCM and persistent $\mathrm{AF}$ show markedly decreased myocardial perfusion at rest and under hyperemia whereas CVR is increased.

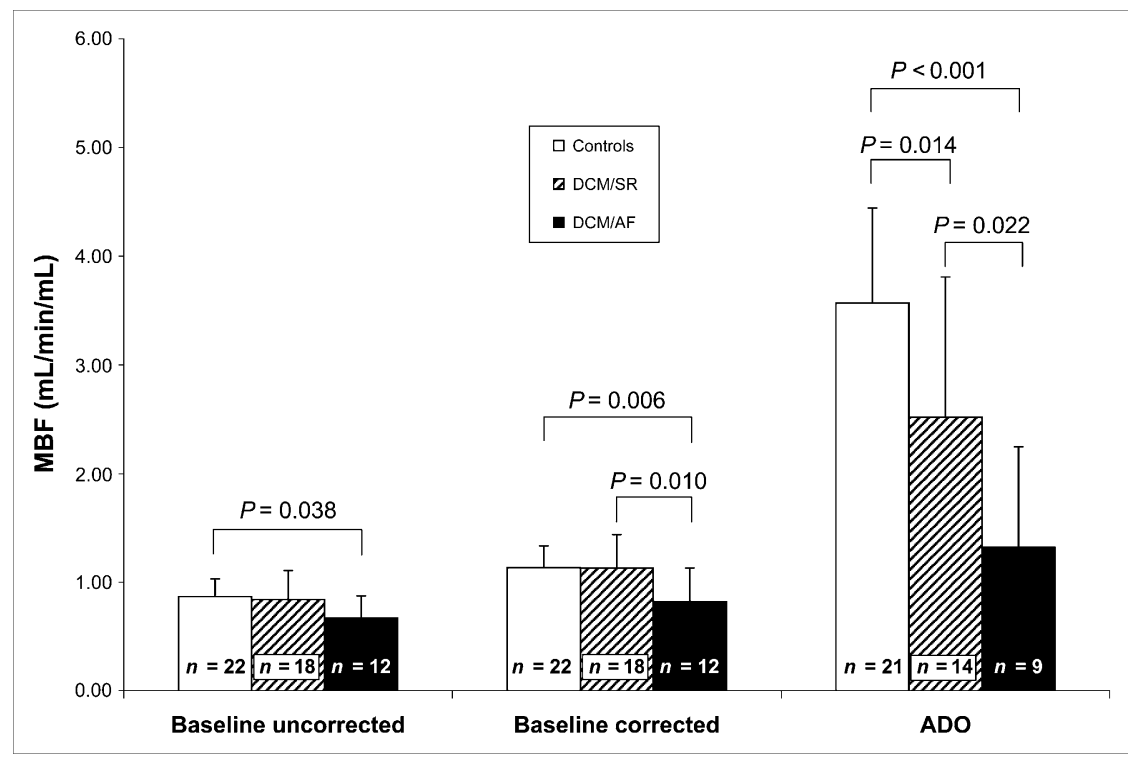

FIGURE 1. MBF in DCM/AF, DCM/SR, and controls as measured by $\mathrm{H}_{2}{ }^{15} \mathrm{O}$ PET at baseline and under adenosine infusion (ADO). MBF at baseline has been corrected for rate-pressure product. 


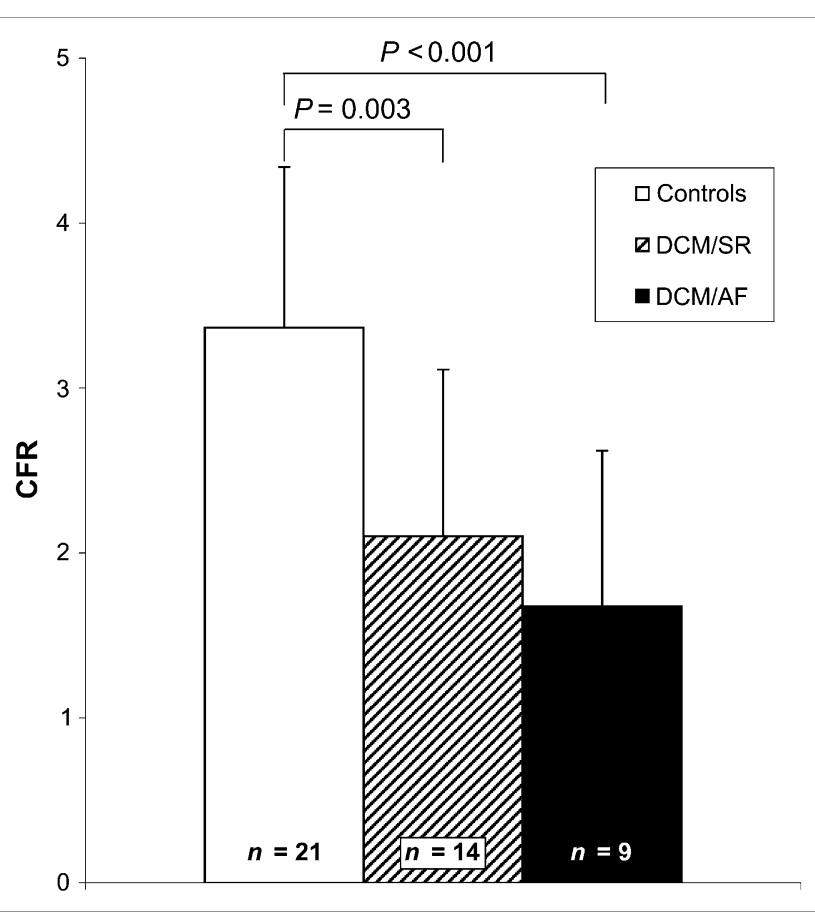

FIGURE 2. Coronary flow reserve (CFR) calculated from MBF under adenosine infusion divided by baseline MBF corrected for rate-pressor product in DCM/AF, DCM/SR, and controls as determined by $\mathrm{H}_{2}{ }^{15} \mathrm{O}$ PET.

\section{Resting Perfusion}

In comparing DCM patients with SR versus controls, we found that the underlying structural heart disease alone did not affect resting MBF or CVR. However, in DCM patients with $\mathrm{AF}$, the resting $\mathrm{MBF}$ corrected for RPP was significantly diminished by about $27 \%$ whereas the CVR was elevated by about $42 \%$. This finding is novel, since other techniques previously investigating perfusion in patients with DCM and AF could not quantify baseline MBF and CVR in absolute terms. The findings are in line with, yet exceed, the former finding of an approximately $20 \%$ reduction of resting $\mathrm{MBF}$ corrected for RPP in idiopathic AF (2).

\section{Resting Perfusion Uncorrected for Rate-Pressure}

Product

Uncorrected MBF was about $20 \%$ lower in patients with DCM and additional AF than in patients with DCM in SR. This finding underlines that differences in myocardial perfusion at baseline do not occur secondary to the correction for the rate-pressure product but already exist in absolute uncorrected data. The fact that correcting for the respective cardiac workload sharpens the contrast between DCM patients with and without AF significantly due to a higher rate-pressure product in DCM/AF patients points to the insufficient pathophysiologic compensatory mechanism. Patients with DCM and additional AF achieve lower myocardial perfusion despite a simultaneously higher cardiac workload than that in DCM patients in SR. Therefore, correction of the MBF at baseline for the rate-pressure product adds relevant information since it reveals the whole dimension of the diminished myocardial perfusion in contrast to a simultaneously elevated (ineffective) cardiac workload in patients with DCM and AF.

\section{Regional Perfusion Distribution}

Our study did not show any divergent regional perfusion patterns in patients with DCM and additional AF, compared with SR. The diminished myocardial perfusion in the DCM/AF group prevails equally in all regions of the heart. This finding supports the hypothesis of a global pathophysiologic mechanism diminishing perfusion in all myocardial regions simultaneously and equally. A regionally localized influencing factor can merely be excluded from these data. This is in line with many mechanistic studies describing systemically changed vasoconstrictive tones in AF.

\section{Hyperemic Perfusion}

Under adenosine infusion, DCM patients with additional AF reached only about $37 \%$ of the MBF measured in controls and about $52 \%$ of the MBF in DCM patients without AF. In comparing DCM patients without AF versus controls, we found that the underlying DCM seemed to have an independent effect on hyperemic MBF, in line with earlier studies (14). However, hyperemic perfusion in DCM

\begin{tabular}{|c|c|c|c|c|c|}
\hline & MBF-RPP septal & MBF-RPP anterior & MBF-RPP lateral & MBF-RPP inferior & $n$ \\
\hline \multicolumn{6}{|l|}{ Controls } \\
\hline Baseline & $1.23 \pm 0.50$ & $1.11 \pm 0.19$ & $1.19 \pm 0.28$ & $1.03 \pm 0.25$ & 22 \\
\hline Adenosine & $3.68 \pm 1.19$ & $3.35 \pm 0.85$ & $3.57 \pm 1.05$ & $3.68 \pm 1.17$ & 22 \\
\hline \multicolumn{6}{|l|}{ DCM/SR } \\
\hline Baseline & $1.07 \pm 0.37$ & $1.09 \pm 0.33$ & $1.30 \pm 0.44$ & $1.14 \pm 0.38$ & 18 \\
\hline Adenosine & $2.75 \pm 1.72$ & $2.37 \pm 1.11$ & $2.49 \pm 1.45$ & $2.65 \pm 1.39$ & 14 \\
\hline \multicolumn{6}{|l|}{ DCM/AF } \\
\hline Baseline & $0.76 \pm 0.33$ & $0.84 \pm 0.25$ & $0.96 \pm 0.45$ & $0.73 \pm 0.33$ & 12 \\
\hline Adenosine & $1.17 \pm 0.65$ & $1.48 \pm 0.97$ & $1.58 \pm 1.21$ & $1.24 \pm 0.96$ & 10 \\
\hline
\end{tabular}




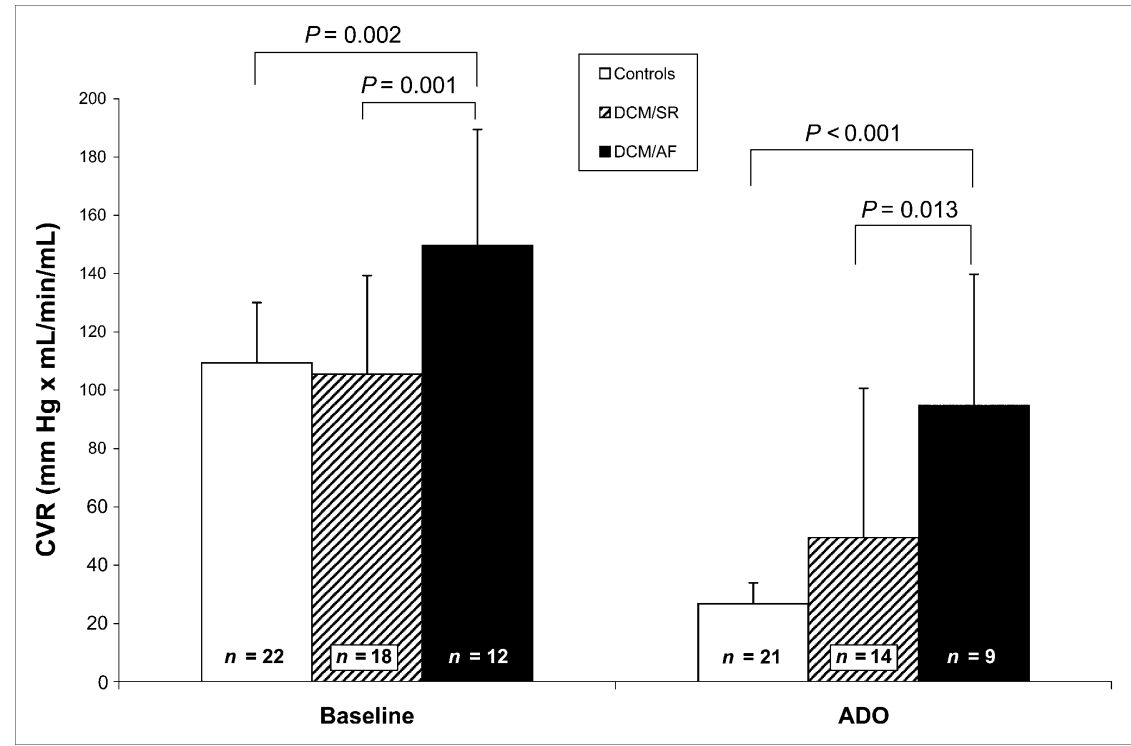

FIGURE 3. CVR at baseline and under adenosine administration (ADO) in DCM/ $\mathrm{AF}, \mathrm{DCM} / \mathrm{SR}$, and controls. patients with AF is massively diminished and does not exceed even levels of normal resting flow within controls. Accordingly, minimal CVR under adenosine was twice as high in DCM patients with AF as in DCM patients without AF. The presence of AF in DCM patients was associated with a degree of perfusion impairment exceeding that found in otherwise healthy hearts (1) or in valvular (15) and artificially induced acute AF (16). To our knowledge, impairments of such an extent in absolute hyperemic perfusion are unparalleled in the literature so far.

\section{Pathophysiologic Considerations}

This study design was, for the first time, able to show perfusion differences between DCM patients with and without AF but prevented us from examining pathophysiologic considerations. This ability would require an interventional design, such as before and after electrical cardioverter therapy. Theoretically, our observations could be attributed to the tachyarrhythmia itself. Besides the acceleration in heart rate, the irregularity of ventricular cycle lengths in AF may be disadvantageous for hemodynamics (17) and overall cardiac function. Intraindividual reduction of variance of cycle length by pacing (18) or cardioversion to SR (19) reameliorated these functional deficits. Still, there was no interindividual correlation between variance of cycle length and myocardial perfusion within our DCM/AF population.

The diminished perfusion was accompanied by an elevation in CVR. This elevation might indicate a shift toward an increased vasoconstrictive tone. Former studies support the hypothesis of a systemically mediated vasoconstriction in $\mathrm{AF}$, showing that forearm vessels also lose vasodilator capacity during AF (20). In DCM patients, however, perfusion abnormalities appear to be restricted to the microvasculature without afflicting larger vessels (21).

Potential mediators of vasoconstriction might be of neurohumoral origin, as suggested by raised plasma levels of atrial natriuretic peptide or brain natriuretic peptide under AF (22), as well as their renormalization after successful catheter ablation of AF (23). Additionally, raised levels of the angiotensin-converting enzyme have been found in chronic AF (24).

A second component might be an elevated sympathetic tone (25) with a reamelioration of hyperemic flow reserve after administration of an $\alpha_{1}$-adrenoceptor antagonist (26). A change in adrenergic tone was also observed for an underlying DCM (27).

Furthermore, the arrhythmia might even join forces with the underlying structural heart disease and remodel the myocardium. Evidence of negative remodeling in AF has been supplied (28), as well as proof of its iterative reversibility (29).

To elucidate how far these observations of impaired myocardial perfusion correlate with outcome in patients with underlying DCM and additional AF, and whether restoration of SR can reameliorate myocardial perfusion, was not in the scope of this study and requires further longitudinal and interventional studies.

\section{Limitations}

This study describes, for the first time, impaired perfusion reserve and increased coronary resistance in DCM and AF. To study whether these changes are associated with, or independent of, the arrhythmia, one could individually reanalyze patients (second PET series) after restoration of SR. However, the patients in this study were recruited on the basis of proven nonischemic DCM. In none of the patients was restoration of SR by electrical cardioverter therapy planned or feasible. This limitation prevents us from drawing the respective pathophysiologic conclusion.

Statistical proof that AF diminishes myocardial perfusion as an independent factor with the help of a multivariate analysis could not be provided because of the small patient sample in combination with a broad variety of potentially confounding factors. 
Patients with DCM/AF were slightly older than DCM patients without AF. However, when tested, this finding was not statistically significant. Furthermore, New York Heart Association functional classes and echocardiographic findings were comparable between the 2 groups; DCM patients therefore seem to be well comparable between the groups.

Although control subjects showed a tendency toward higher cardiovascular risk as assessed by analysis of risk factors known for their detrimental effects on myocardial perfusion, there was no statistically significant difference between the 2 DCM groups. However, this could result only in lower differences between controls and DCM patients and does not explain the findings of the study. Because of the relatively small sample size, potential effects of digitalis could not be analyzed statistically. There have been hints that cardiac glycosides (especially ouabain) cause acute vasoconstriction that fades within minutes (30). In contrast, for digoxin and digitoxin, which were given to our patients, vasodilatory effects have been discussed (31). In early invasive studies, the resulting absolute global MBF was found to be unchanged by cardiac glycosides (32). Especially in the long-term treatment of failing human hearts, beneficial effects on coronary circulation can be expected, as concluded in one study (33). Therefore, it is unlikely that the effects of digitalis contributed to our findings of diminished perfusion in additional AF.

\section{CONCLUSION}

Patients with nonischemic DCM and persistent AF, compared with those having SR, show significantly diminished myocardial perfusion and perfusion reserve and increased CVR. Further studies on the pathophysiologic interrelation between the arrhythmia and this functional finding are warranted.

\section{ACKNOWLEDGMENTS}

We gratefully acknowledge the assistance and support of Silke Schroer and of the PET Centre Group. This study was supported by grants from the Interdisciplinary Centre of Clinical Research Münster (IZKF, project B10) and from the Deutsche Forschungsgemeinschaft (DFG SFB 656, projects $\mathrm{A} 1$ and $\mathrm{C} 2$ ).

\section{REFERENCES}

1. Kannel WB, Benjamin EJ. Status of the epidemiology of atrial fibrillation. Med Clin North Am. 2008;92:17-40.

2. Range FT, Schäfers M, Acil T, et al. Impaired myocardial perfusion and perfusion reserve associated with increased coronary resistance in persistent idiopathic atrial fibrillation. Eur Heart J. 2007;28:2223-2230.

3. Haissaguerre M, Bonnet J, Billes MA, et al. Prevalence, signification and prognosis of auricular arrhythmia in dilated myocardiopathies: apropos of 236 cases. Arch Mal Coeur Vaiss. 1985;78:536-541.

4. Khan IA, Tun A, Wattanasauwan N, et al. Elevation of serum cardiac troponin $\mathrm{I}$ in noncardiac and cardiac diseases other than acute coronary syndromes. Am J Emerg Med. 1999;17:225-229.

5. Hagens VE, Crijns HJ, Van Veldhuisen DJ, et al. Rate control versus rhythm control for patients with persistent atrial fibrillation with mild to moderate heart failure: results from the RAte Control versus Electrical cardioversion (RACE) study. Am Heart J. 2005;149:1106-1111.
6. Hsu LF, Jais P, Sanders P, et al. Catheter ablation for atrial fibrillation in congestive heart failure. N Engl J Med. 2004;351:2373-2383.

7. Neglia D, Parodi O, Gallopin M, et al. Myocardial blood flow response to pacing tachycardia and to dipyridamole infusion in patients with dilated cardiomyopathy without overt heart failure: a quantitative assessment by positron emission tomography. Circulation. 1995;92:796-804.

8. Neglia D, Michelassi C, Trivieri MG, et al. Prognostic role of myocardial blood flow impairment in idiopathic left ventricular dysfunction. Circulation. 2002;105:186-193.

9. Diamond GA, Forrester JS. Analysis of probability as an aid in the clinical diagnosis of coronary artery disease. N Engl J Med. 1979;300:1350-1358.

10. Pop GA, Meeder HJ, Roelandt JR, et al. Transthoracic echo/Doppler in the identification of patients with chronic non-valvular atrial fibrillation at risk for thromboembolic events. Eur Heart J. 1994;15:1545-1551.

11. Kaufmann PA, Gnecchi-Ruscone T, Yap JT, Rimoldi O, Camici PG. Assessment of the reproducibility of baseline and hyperemic myocardial blood flow measurements with oxygen-15-labeled water and PET. J Nucl Med. 1999;40:1848-1856.

12. Schäfers KP, Spinks TJ, Camici PG, et al. Absolute quantification of myocardial blood flow with $\mathrm{H}_{2}{ }^{15} \mathrm{O}$ and 3-dimensional PET: an experimental validation. J Nucl Med. 2002;43:1031-1040.

13. Campisi R, Czernin J, Schoder H, et al. Effects of long-term smoking on myocardial blood flow, coronary vasomotion, and vasodilator capacity. Circulation. 1998;98:119-125.

14. Inoue T, Sakai Y, Morooka S, et al. Coronary flow reserve in patients with dilated cardiomyopathy. Am Heart J. 1993;125:93-98.

15. Kochsiek K, Tauchert M, Heiss W, Strauer BE, Sonntag H. Influence of auricular fibrillation on the coronary reserve: studies before and following cardioversion. Verh Dtsch Ges Kreislaufforsch. 1971;37:326-330.

16. Kochiadakis GE, Skalidis EI, Kalebubas MD, et al. Effect of acute atrial fibrillation on phasic coronary blood flow pattern and flow reserve in humans. Eur Heart J. 2002;23:734-741.

17. Clark DM, Plumb VJ, Epstein AE, Kay GN. Hemodynamic effects of an irregular sequence of ventricular cycle lengths during atrial fibrillation. $J \mathrm{Am}$ Coll Cardiol. 1997;30:1039-1045.

18. Lau CP, Leung WH, Wong CK, Tai YT, Cheng CH. A new pacing method for rapid regularization and rate control in atrial fibrillation. Am J Cardiol. 1990;65:1198-1203.

19. Morris JJ Jr, Entman M, North WC, Kong Y, Mcintosh H. The changes in cardiac output with reversion of atrial fibrillation to sinus rhythm. Circulation. 1965;31: 670-678.

20. Takahashi N, Ishibashi Y, Shimada T, et al. Atrial fibrillation impairs endothelial function of forearm vessels in humans. J Card Fail. 2001;7:45-54.

21. Stolen KQ, Kemppainen J, Kalliokoski KK, et al. Myocardial perfusion reserve and oxidative metabolism contribute to exercise capacity in patients with dilated cardiomyopathy. J Card Fail. 2004;10:132-140.

22. Parthenakis FI, Patrianakos AP, Skalidis EI, et al. Atrial fibrillation is associated with increased neurohumoral activation and reduced exercise tolerance in patients with non-ischemic dilated cardiomyopathy. Int J Cardiol. 2007;118:206-214.

23. Yamada T, Murakami Y, Okada T, et al. Plasma atrial natriuretic peptide and brain natriuretic peptide levels after radiofrequency catheter ablation of atrial fibrillation. Am J Cardiol. 2006;97:1741-1744.

24. Goette A, Staack T, Röcken C, et al. Increased expression of extracellular signalregulated kinase and angiotensin-converting enzyme in human atria during atrial fibrillation. J Am Coll Cardiol. 2000;35:1669-1677.

25. Wasmund SL, Li JM, Page RL, et al. Effect of atrial fibrillation and an irregular ventricular response on sympathetic nerve activity in human subjects. Circulation. 2003;107:2011-2015.

26. Ertl G, Wichmann J, Kaufmann M, Kochsiek K. Alpha-receptor constriction induced by atrial fibrillation during maximal coronary dilatation. Basic Res Cardiol. 1986;81:29-39.

27. Erdogan D, Gullu H, Caliskan M, et al. Nebivolol improves coronary flow reserve in patients with idiopathic dilated cardiomyopathy. Heart. 2007;93:319-324.

28. Nattel S. New ideas about atrial fibrillation 50 years on. Nature. 2002;415:219-226.

29. Reant $P$, Lafitte $S$, Jais $P$, et al. Reverse remodeling of the left cardiac chambers after catheter ablation after 1 year in a series of patients with isolated atrial fibrillation. Circulation. 2005;112:2896-2903.

30. Vatner SF, Higgins CB, Franklin D, Braunwald E. Effects of a digitalis glycoside on coronary and systemic dynamics in conscious dogs. Circ Res. 1971;28:470-479.

31. Kirch W. Pressor and vascular effects of cardiac glycosides. Eur J Clin Invest. 2001;31:5-9.

32. Bing RJ, Maraist FM, Dammann JF Jr, et al. Effect of strophanthus on coronary blood flow and cardiac oxygen consumption of normal and failing human hearts. Circulation. 1950;2:513-516.

33. Longhurst JC, Ross J Jr. Extracardiac and coronary vascular effects of digitalis. J Am Coll Cardiol. 1985;5:99A-105A. 